\title{
ON THE CLASSIFICATION OF CONTACT RIEMANNIAN MANIFOLDS SATISFYING THE CONDITION (C)
}

\author{
JONG TAEK CHO \\ Department of Mathematics, Chonnam National University, The Institute of Basic Sciences, \\ Kwangju 500-757, Korea \\ e-mail: jtcho@chonnam.ac.kr \\ and SUN HYANG CHUN \\ Department of Mathematics, Graduate School Chonnam National University, Kwangju 500-757, Korea
}

(Received 1 May, 2002; accepted 3 October, 2002)

\begin{abstract}
Given a contact form $\eta$, there is a one-to-one correspondence between the Riemannian structures $(\eta, g)$ and the CR-structures $(\eta, L)$. It is interesting to study the interaction between the two associated structures. We approach the geometry of contact Riemannian manifolds in connection with their associated CR-structures. In this context, for a contact Riemannian manifold $(M ; \eta, g)$ we consider the Jacobi-type operator $R_{\dot{\gamma}}=R(\cdot, \dot{\gamma}) \dot{\gamma}$ along a self-parallel curve $\gamma$ with respect to the (generalized) Tanaka connection $\hat{\nabla}$.
\end{abstract}

2000 Mathematics Subject Classification. 53C15, 53C25.

1. Introduction. The contact structure $\eta$ is a global differentiable one-form on a smooth manifold $M^{2 n+1}$ such that $\eta \wedge(d \eta)^{n} \neq 0$ everywhere on $M$. It is well-known that there exists an associated Riemannian structure (metric) $g$ and a (1,1)-type tensor $\phi$, where $g$ and $\phi$ are canonically related. We call the pair $(\eta, g)$ a contact Riemannian structure and $M=(M ; \eta, g)$ a contact Riemannian manifold. S. Sasaki and Y. Hatakeyama [22] defined the normality of the contact Riemannian structure (see section 2). A normal contact Riemannian manifold is said to be a Sasakian manifold. In [19] it was proved that a Sasakian manifold which is locally symmetric $(\nabla R=0)$ must have constant curvature +1 , where $\nabla$ is the Levi-Civita connection. This fact means that local symmetry is a very strong condition for a Sasakian manifold. For this reason, T. Takahashi ([24]) introduced the notion of Sasakian locally $\phi$-symmetric spaces which may be considered as the analogues of locally Hermitian symmetric spaces. A contact Riemannian locally $\phi$-symmetric space is defined as a generalization of a Sasakian locally $\phi$-symmetric space and investigated in [5].

One the other hand, the associated CR-structure of a given contact Riemannian manifold $M=(M ; \eta, g)$ is given by the holomorphic subbundle

$$
\mathscr{H}=\{X-i \bar{\phi} X: X \in D\}
$$

of the complexification $T M^{\mathbb{C}}$ of the tangent bundle $T M$, where $D$ is the subbundle of $T M$ defined by the kernel of $\eta$ and $\bar{\phi}=\phi \mid D$, the restriction of $\phi$ to $D$. Then we see that

This work was financially supported by Chonnam National University in the program, 2001. 
each fibre $\mathscr{H}_{x}(x \in M)$ is of complex dimension $n$ and $\mathscr{H} \cap \overline{\mathscr{H}}=\{0\}$. Furthermore, we have $\mathbb{C} D=\mathscr{\mathscr { H }} \oplus \overline{\mathscr{H}}$. We say that the associated $\mathrm{CR}$-structure is integrable if $[\mathscr{H}, \mathscr{\mathscr { H }}] \subset$ $\mathscr{H}$. For $\mathscr{H}$ we define the Levi form by

$$
L: D \times D \rightarrow \mathscr{F}(M), \quad L(X, Y)=-d \eta(X, \phi Y)
$$

where $\mathscr{F}(M)$ denotes the algebra of differential functions on $M$. Then we see that the Levi form is Hermitian and positive definite, that is, the CR-structure is strongly pseudo-convex, pseudo-Hermitian CR-structure. In fact, for a contact manifold $(M ; \eta)$, there is a correspondence between the contact Riemannian structure $(\eta, g)$ and strongly pseudo-convex, pseudo-Hermitian CR-structure $(\eta, L)$ by the relation $g=L+\eta \otimes \eta$, where we denote by the same letter $L$ the natural extension of the Levi form to a $(0,2)$-tensor field on $M$. N. Tanaka [25] defined the canonical affine connection on a nondegenerate integrable CR-manifold. In [27] S. Tanno defined the generalized Tanaka connection $\hat{\nabla}$ on a contact Riemannian manifold and further, he proved that for a given contact Riemannian manifold $M$ the associated CR-structure is strongly pseudo-convex integrable if and only if $M$ satisfies the integrability condition $Q=0$ (see section 2 ), in which case the connection $\hat{\nabla}$ coincides with the Tanaka connection. Here, we note that the normality of a contact Riemannian structure implies the integrability of the associated CR-structure, but the converse does not always hold. The associated CR-structures of 3-dimensional contact Riemannian manifolds are always integrable (see [27]). Also, we see that their associated CR-structures are integrable for (contact Riemannian) $(k, \mu)$-spaces (see [2], [6] or [12]).

It is interesting to study the geometry of a given contact Riemannian manifold $(M ; \eta, g)$ in connection with the associated CR-structure, particularly with the generalized Tanaka connection. In this context, we define the Jacobi-type operator $R_{\dot{\gamma}}=R(\cdot, \dot{\gamma}) \dot{\gamma}$ along a unit $\hat{\nabla}$-geodesic $\gamma$. Here, we observe that the geodesics of the Levi-Civita connection and the generalized Tanaka connection do not coincide in general. In the preceding paper [12] the first author has introduced a new class of contact Riemannian manifolds satisfying the condition (C), i.e., the Jacobi-type operator field $R_{\dot{\gamma}}$ is diagonalizable by a $\hat{\nabla}$-parallel orthonormal frame field along $\gamma$ and its eigenvalues are constant along $\gamma$, or equivalently,

$$
\left(\hat{\nabla}_{\dot{\gamma}} R\right)(\cdot, \dot{\gamma}) \dot{\gamma}=0
$$

for any unit $\hat{\nabla}$-geodesic $\gamma$, where $\hat{\nabla}$ is the generalized Tanaka connection. Further, in [12] it has been shown that $(k, 2)$-spaces $(k \neq 1)$, including the standard contact Riemannian structure of the unit tangent sphere bundle $T_{1} M$ of $M$ with constant curvature -1 , are examples that are neither Sasakian nor locally symmetric but satisfy the condition (C) for any $\hat{\nabla}$-geodesic $\gamma$. Also, it is remarkable that a $(k, \mu)$-space with $k=\mu=0$ of dimension $\geq 5$, which is a product of $(n+1)$-dimensional flat manifold and $n$-dimensional space of constant curvature 4 , is locally symmetric but $M$ fails to satisfy the condition (C) for any $\hat{\nabla}$-geodesic $\gamma$. Continuing the preceding work, in this paper we develop further the results in [12]. More precisely, in section 3 we prove

Theorem A. Let $M$ be a $(k, \mu)$-space. Then $M$ satisfies the condition (C) for any $\hat{\nabla}$-geodesic $\gamma$ if and only if (1) $k=1$ and $M$ is Sasakian locally $\phi$-symmetric or (2) $\mu=0$ in which case $M$ is 3-dimensional, or (3) $\mu=2$. 
In [12] it was also proved that the standard contact Riemannian structure of the unit tangent sphere bundle $T_{1} M$ of a 2-dimensional Riemannian manifold $M$ satisfies condition (C) for any $\hat{\nabla}$-geodesic $\gamma$ if and only if $M$ has constant Gauss curvature 1, 0 or -1 . In section 4 , we prove this result for arbitrary dimension. Namely, we prove

TheOREM B. Let $M$ be a $(n+1)$-dimensional Riemannian manifold. Then the standard contact Riemannian structure of the unit tangent sphere bundle $T_{1} M$ satisfies the condition (C) for any $\hat{\nabla}$-geodesic $\gamma$ if and only if the base manifold $M$ is of constant curvature $c=1, n=1$ and $c=0$, or $c=-1$.

REMARK 1. Recently, it was proved in [4] ([20] and [21], respectively) that the base manifold is of constant curvature $c=-1, c=1(c=0, c=1$, respectively) if and only if the standard contact Riemannian structure on the unit tangent sphere bundle is a critical point of some functional on the set of associated Riemannian metrics $\mathscr{M}(\eta)$ of a given contact form $\eta$.

Finally, in section 5, we give a local and a global classification of 3-dimensional contact Riemannian manifolds satisfying the condition (C) for any $\hat{\nabla}$-geodesic $\gamma$. More precisely, we prove

THEOREM C (local classification). Let $M$ be a 3-dimensional contact Riemannian manifold. Then $M$ satisfies the condition (C) for any $\hat{\nabla}$-geodesic $\gamma$ if and only if $M$ is locally isometric to one of the following spaces:

(1) a Sasakian $\phi$-symmetric space;

(2) $S U(2)$ (or $S O(3)), S L(2, \mathbb{R})($ or $O(1,2))$ with a special left-invariant contact metric which is not Sasakian, respectively;

(3) a flat manifold.

In [7] the authors gave a classification of Sasakian $\phi$-symmetric spaces (complete and simply connected Sasakian locally $\phi$-symmetric spaces). Together with this classification we have

THEOREM D (global classification). Let $M$ be a complete and simply connected 3-dimensional contact Riemannian manifold. Then $M$ satisfies the condition (C) for any $\hat{\nabla}$-geodesic $\gamma$ if and only if $M$ is isometric to one of the following spaces:

(1) the standard unit sphere $S^{3} ; S U(2), \widehat{S L(2, \mathbb{R})}$ (the universal covering of $S L(2, \mathbb{R})$ ) or the Heisenberg group $H$ with a left-invariant Sasakian metric, respectively;

(2) $S U(2), S \widetilde{S L(2, \mathbb{R})}$ with a special left-invariant contact metric which is not Sasakian, respectively;

(3) $\mathbb{R}^{3}$.

2. Preliminaries. We start by collecting some fundamental material about contact Riemannian geometry and refer to [2] for further details. All manifolds in the present paper are assumed to be connected and of class $C^{\infty}$.

A $(2 n+1)$-dimensional manifold $M^{2 n+1}$ is said to be a contact manifold if it admits a global one-form $\eta$ such that $\eta \wedge(d \eta)^{n} \neq 0$ everywhere. Given a contact form $\eta$, there exists a unique vector field $\xi$, called the characteristic vector field, satisfying $\eta(\xi)=1$ and $d \eta(\xi, X)=0$ for any vector field $X$. It is well-known that there also exists a Riemannian metric $g$ and a $(1,1)$-tensor field $\phi$ such that

$$
\eta(X)=g(X, \xi), \quad d \eta(X, Y)=g(X, \phi Y), \quad \phi^{2} X=-X+\eta(X) \xi,
$$


where $X$ and $Y$ are vector fields on $M$. From (2.1), it follows that

$$
\phi \xi=0, \quad \eta \circ \phi=0, \quad g(\phi X, \phi Y)=g(X, Y)-\eta(X) \eta(Y) .
$$

A Riemannian manifold $M$ equipped with structure tensors $(\eta, g)$ satisfying $(2.1)$ is said to be a contact Riemannian manifold or contact metric manifold and it is denoted by $M=(M ; \eta, g)$. Given a contact Riemannian manifold $M$, we define a $(1,1)$-tensor field $h$ by $h=\frac{1}{2} L_{\xi} \phi$, where $L$ denotes Lie differentiation. Then we may observe that $h$ is symmetric and satisfies

$$
\begin{gathered}
h \xi=0, \quad h \phi=-\phi h \\
\nabla_{X} \xi=-\phi X-\phi h X
\end{gathered}
$$

where $\nabla$ is Levi-Civita connection. From (2.3) and (2.4), we see that each trajectory of $\xi$ is a geodesic. We denote by $R$ the Riemannian curvature tensor defined by

$$
R(X, Y) Z=\nabla_{X}\left(\nabla_{Y} Z\right)-\nabla_{Y}\left(\nabla_{X} Z\right)-\nabla_{[X, Y]} Z
$$

for all vector fields $X, Y, Z$. Along a trajectory of $\xi$, the Jacobi operator $R_{\xi}=R(\cdot, \xi) \xi$ is a symmetric $(1,1)$-tensor field. We have

$$
\begin{gathered}
\left(\operatorname{trace} R_{\xi}\right)=g(S \xi, \xi)=2 n-\left(\operatorname{trace} h^{2}\right), \\
R_{\xi}=\phi R_{\xi} \phi-2\left(h^{2}+\phi^{2}\right), \\
\nabla_{\xi} h=\phi-\phi R_{\xi}-\phi h^{2}, \\
g(R(X, Y) \xi, Z)=g\left(\left(\nabla_{Y} \phi\right) X-\left(\nabla_{X} \phi\right) Y, Z\right)+g\left(\left(\nabla_{Y} \phi h\right) X-\left(\nabla_{X} \phi h\right) Y, Z\right)
\end{gathered}
$$

for all vector fields $X, Y, Z$ on $M$, where $S$ is the Ricci $(1,1)$-tensor on $M$. A contact Riemannian manifold for which $\xi$ is a Killing vector field is called a $K$-contact manifold. It is easy to see that a contact Riemannian manifold is $K$-contact if and only if $h=0$. For a contact Riemannian manifold $M$, one may define naturally an almost complex structure $J$ on $M \times \mathbb{R}$ by

$$
J\left(X, f \frac{d}{d t}\right)=\left(\phi X-f \xi, \eta(X) \frac{d}{d t}\right),
$$

where $X$ is a vector field tangent to $M, t$ the coordinate of $\mathbb{R}$ and $f$ a function on $M \times \mathbb{R}$. If the almost complex structure $J$ is integrable, $M$ is said to be normal or Sasakian. It is known that $M$ is normal if and only if $M$ satisfies

$$
[\phi, \phi]+2 d \eta \otimes \xi=0,
$$

where $[\phi, \phi]$ is the Nijenhuis torsion of $\phi$. A Sasakian manifold is also characterized by the condition

$$
\left(\nabla_{X} \phi\right) Y=g(X, Y) \xi-\eta(Y) X
$$

for all vector fields $X$ and $Y$ on the manifold and this is equivalent to

$$
R(X, Y) \xi=\eta(Y) X-\eta(X) Y
$$

for all vector fields $X$ and $Y$. 
For a contact Riemannian manifold $M$, the tangent space $T_{p} M$ of $M$ at each point $p \in M$ is decomposed as $T_{p} M=D_{p} \oplus\{\xi\}_{p}$ (direct sum), where we denote $D_{p}=\left\{v \in T_{p} M \mid \eta(v)=0\right\}$. Then $D: p \rightarrow D_{p}$ defines a distribution orthogonal to $\xi$. The $2 n$-dimensional distribution $D$ is called the contact distribution. We see that the restriction $\bar{\phi}=\phi \mid D$ of $\phi$ to $D$ defines an almost complex structure on $D$, and furthermore that the associated Levi form, which is defined by $L(X, Y)=-d \eta(X, \bar{\phi} Y)$, $X, Y \in D$, is positive definite and Hermitian. We call the pair $(\eta, \bar{\phi})$ a strongly pseudoconvex, pseudo-hermitian structure on $M$. Since $d \eta(\phi X, \phi Y)=d \eta(X, Y)$, we see that $[\bar{\phi} X, \bar{\phi} Y]-[X, Y] \in D$ for $X, Y \in D$. Further if $M$ satisfies the condition

$$
[\bar{\phi}, \bar{\phi}](X, Y)=0
$$

for $X, Y \in D$, then the pair $(\eta, \bar{\phi})$ is called a strongly pseudo-convex integrable $C R$ structure, (associated with the contact Riemannian structure $(\eta, g)$ ). Taking account of (2.9) we see that for a Sasakian manifold the associated CR-structure is strongly pseudo-convex integrable (cf. [16]).

Now, we review the generalized Tanaka connection ([27]) on a contact Riemannian manifold $M=(M ; \eta, g)$. The generalized Tanaka connection $\hat{\nabla}$ is defined by

$$
\hat{\nabla}_{X} Y=\nabla_{X} Y+\eta(X) \phi Y+\left(\nabla_{X} \eta\right)(Y) \xi-\eta(Y) \nabla_{X} \xi
$$

for all vector fields $X$ and $Y$ on $M$. Together with (2.4), $\hat{\nabla}$ may be rewritten as

$$
\hat{\nabla}_{X} Y=\nabla_{X} Y+\eta(X) \phi Y+\eta(Y)(\phi X+\phi h X)-g(\phi X+\phi h X, Y) \xi
$$

and we see that the generalized Tanaka connection $\hat{\nabla}$ has the torsion $\hat{T}(X, Y)=$ $2 g(X, \phi Y) \xi+\eta(Y) \phi h X-\eta(X) \phi h Y$. We put

$$
A(X, Y)=\eta(X) \phi Y+\eta(Y)(\phi X+\phi h X)-g(\phi X+\phi h X, Y) \xi
$$

for all vector fields $X$ and $Y$ on $M$. Then $A$ is a (1,2)-tensor field on $M$ and $\hat{\nabla}_{X} Y=$ $\nabla_{X} Y+A(X, Y)$. In particular, for a $K$-contact Riemannian manifold we get

$$
A(X, Y)=\eta(X) \phi Y+\eta(Y) \phi X-g(\phi X, Y) \xi,
$$

where $X$ and $Y$ are vector fields. For a given contact Riemannian manifold $M$ the associated CR-structure is strongly pseudo-convex integrable if and only if $M$ satisfies the integrability condition $Q=0$, where $Q$ is a $(1,2)$-tensor field on $M$ defined by

$$
Q(X, Y)=\left(\nabla_{X} \phi\right) Y-g(X+h X, Y) \xi+\eta(Y)(X+h X)
$$

for all vector fields $X, Y$ on $M$ (see [27, Proposition 2.1]). Further, the following result was proved.

Proposition 2.1 ([27]). The generalized Tanaka connection $\hat{\nabla}$ on a contact Riemannian manifold $M=(M ; \eta, g)$ is the unique linear connection satisfying the following conditions:

(i) $\hat{\nabla} \eta=0, \hat{\nabla} \xi=0$;

(ii) $\hat{\nabla} g=0$;

(iii(a)) $\hat{T}(X, Y)=2 d \eta(X, Y) \xi, X, Y \in D$;

(iii(b)) $\hat{T}(\xi, \phi Y)=-\phi \hat{T}(\xi, Y), Y \in D$;

(iv) $\left(\hat{\nabla}_{X} \phi\right) Y=Q(X, Y), X, Y \in T M$. 
The Tanaka connection ([25]) on a nondegenerate integrable CR-manifold is defined as the unique linear connection satisfying (i), (ii), (iii(a)), (iii(b)) and $\hat{\nabla} \phi=0$. The metric affine connection $\hat{\nabla}$ is a natural and proper generalization of the Tanaka connection. In fact, in [1] the authors deal with the use of $\hat{\nabla}$ in the non-integrable case.

Let $\gamma$ be a $\hat{\nabla}$-geodesic parametrized by the arc-length parameter $s$, where a $\hat{\nabla}$ geodesic means a geodesic with respect to $\hat{\nabla}$. From $(2.11)$ we see that a $\hat{\nabla}$-geodesic does not coincide with a $\nabla$-geodesic. Define the Jacobi operator $R_{\dot{\gamma}}$ by $R_{\dot{\gamma}}=R(\cdot, \dot{\gamma}) \dot{\gamma}$ along $\gamma$, where $\dot{\gamma}$ is the unit tangent vector field of $\gamma$. Then $R_{\dot{\gamma}}$ is a symmetric $(1,1)$-tensor field along $\gamma$. Moreover, from (i) of Proposition 2.1 we observe that $\eta(\dot{\gamma})$ is constant along $\gamma$, and thus a $\hat{\nabla}$-geodesic whose tangent initially belongs to $D$ remains in $D$. We call such a $\hat{\nabla}$-geodesic which is tangent to $D$ a horizontal $\hat{\nabla}$-geodesic.

We recall the definition of a Sasakian locally $\phi$-symmetric space ([24]).

Definition 2.2. A Sasakian manifold $M=(M ; \eta, g)$ is said to be locally $\phi$ symmetric if $\phi^{2}\left(\nabla_{V} R\right)(X, Y) Z=0$ for all vector fields $V, X, Y, Z \in D$.

As a generalization of the above Sasakian one, a contact Riemannian locally $\phi$ symmetric space is defined in [5] by the same condition and is called ([8]) a locally $\phi$-symmetric space in the weak sense. In [12] we have the following characterization of a Sasakian locally $\phi$-symmetric space.

THeOREM 2.3. A Sasakian manifold $M$ is locally $\phi$-symmetric if and only if $M$ satisfies the condition (C) for any horizontal $\hat{\nabla}$-geodesic $\gamma$, or if and only if $M$ satisfies the condition (C) for any $\hat{\nabla}$-geodesic $\gamma$.

3. A contact Riemannian $(k, \mu)$-space. In [6], the $(k, \mu)$-nullity distribution of a contact Riemannian manifold $M$, for the pair $(k, \mu) \in \mathbb{R}^{2}$, is defined by

$$
\begin{aligned}
N(k, \mu): p \rightarrow N_{p}(k, \mu)= & \left\{z \in T_{p} M \mid R(x, y) z=k(g(y, z) x-g(x, z) y)\right. \\
& \left.+\mu(g(y, z) h x-g(x, z) h y) \text { for any } x, y \in T_{p} M\right\} .
\end{aligned}
$$

$\mathrm{A}(k, \mu)$-space is a contact Riemannian manifold with $\xi$ belonging to the $(k, \mu)$-nullity distribution, that is,

$$
R(X, Y) \xi=k(\eta(Y) X-\eta(X) Y)+\mu(\eta(Y) h X-\eta(X) h Y) .
$$

It was shown in [6] that the $(k, \mu)$-spaces are invariant under a $D$-homothetic deformation. As mentioned in the introduction, the associated CR-structures of the $(k, \mu)$-spaces are integrable, that is, $Q=0$. This class contains Sasakian manifolds $(k=1$ and $h=0)$. The unit tangent sphere bundle is a $(k, \mu)$-space if and only if the base manifold is of constant curvature $c$ with $k=c(2-c)$ and $\mu=-2 c([6])$. (By virtue of the result of Y. Tashiro [29], we know that for $c \neq 1$, the unit tangent sphere bundle is non-Sasakian.) Very recently, E. Boeckx [9] presented explicit examples for all possible dimensions and all possible $(k, \mu)$.

THEOREM 3.1 ([12]). Let $M$ be a $(k, \mu)$-space. If $M$ satisfies the condition (C) for any $\hat{\nabla}$-geodesic $\gamma$, then we have:

(i) $k=1$ and $M$ is a Sasakian locally $\phi$-symmetric space;

(ii) $\mu=0$ and $M$ is a 3-dimensional locally $\phi$-symmetric space in the weak sense;

(iii) $\mu=2$ and $M$ is a locally $\phi$-symmetric space in the weak sense. 
In [8] it has been proved that all $(k, \mu)$-spaces are locally $\phi$-symmetric in the strong sense, i.e., the characteristic reflections are local isometries, and hence also in the weak sense. Thus, we have

Proposition 3.2. $A(k, \mu)$-space $(k<1)$ is locally $\phi$-symmetric in the weak sense.

Therefore, together with Proposition 3.2 and the proof of Theorem 3.1 (see [12]), we have Theorem A.

REMARK 2. An example of a contact flat Riemannian structure on $\mathbb{R}^{3}\left(x^{1}, x^{2}, x^{3}\right)$ is given by $\eta=\frac{1}{2}\left(\cos x^{3} d x^{1}+\sin x^{3} d x^{2}\right)$ and $g_{i j}=\frac{1}{4} \delta_{i j}$. For dimension at least 5 a contact manifold cannot admit a contact Riemannian structure of vanishing curvature (cf. [2]). Also, it was proved that a contact Riemannian manifold $M^{2 n+1}$ which satisfy $R(X, Y) \xi=0$ for all vector fields $X$ and $Y$ (i.e., $\xi$ belonging to the $(0,0)$-nullity distribution) is locally a product of a flat $(n+1)$-dimensional manifold and an $n$ dimensional manifold of positive constant sectional curvature equal to 4 . Hence, we see that a contact Riemannian manifold $M^{2 n+1}(n \geq 2)$ satisfying $R(X, Y) \xi=0$ is locally symmetric but it does not satisfy the condition (C) for any $\hat{\nabla}$-geodesic.

4. The unit tangent sphere bundles. The basic facts and fundamental formulas about tangent bundles are well-known (cf. [13], [17], [32]). We briefly review of notations and their definitions. Let $M=(M, G)$ be a $(n+1)$-dimensional Riemannian manifold and $T M$ denote its tangent bundle with the projection $\pi: T M \rightarrow M, \pi(x, u)=x$. For a vector $X \in T_{x} M$, we denote by $X^{H}$ and $X^{V}$, the horizontal lift and the vertical lift, respectively. Then we can define a Riemannian metric $\tilde{g}$, Sasaki metric, on TM in a natural way. That is,

$$
\tilde{g}\left(X^{H}, Y^{H}\right)=\tilde{g}\left(X^{V}, Y^{V}\right)=G(X, Y) \circ \pi, \quad \tilde{g}\left(X^{H}, Y^{V}\right)=0
$$

for all vector fields $X$ and $Y$ on $M$. Also, a natural almost complex structure tensor $J$ of $T M$ is defined by $J X^{H}=X^{V}$ and $J X^{V}=-X^{H}$. Then we easily see that $(T M ; \tilde{g}, J)$ is an almost Hermitian manifold. We note that $J$ is integrable if and only if $(M, G)$ is locally flat ([17]). Now we consider the unit tangent sphere bundle $\left(T_{1} M, g^{\prime}\right)$, which is isometrically embedded hypersurface in $(T M, \tilde{g})$ with unit normal vector field $N=u^{V}$. For $X \in T_{x} M$, we define the tangential lift of $X$ to $(x, u) \in T_{1} M$ by

$$
X_{(x, u)}^{T}=X_{(x, u)}^{V}-G(X, u) N_{(x, u)} .
$$

Clearly, the tangent space $T_{(x, u)} T_{1} M$ spanned by vectors of the form $X^{H}$ and $X^{T}$ where $X \in T_{x} M$. We put

$$
\xi^{\prime}=-J N, \quad \phi^{\prime}=J-\eta^{\prime} \otimes N .
$$

Then we find $g^{\prime}\left(X, \phi^{\prime} Y\right)=2 d \eta^{\prime}(X, Y)$. By taking $\xi=2 \xi^{\prime}, \eta=\frac{1}{2} \eta^{\prime}, \phi=\phi^{\prime}$, and $g=$ $\frac{1}{4} g^{\prime}$, we get the standard contact Riemannian structure $(\phi, \xi, \eta, g)$. Indeed, we easily check these tensors satisfy (2.1). Here, we notice that $\xi$ determines the geodesic flow. The tensors $\xi$ and $\phi$ are explicitly given by

$$
\begin{aligned}
\xi & =2 u^{H}, \\
\phi X^{T} & =-X^{H}+1 / 2 G(X, u) \xi, \\
\phi X^{H} & =X^{T}
\end{aligned}
$$


where $X$ and $Y$ are vector fields on $M$. From now, we consider $T_{1} M=\left(T_{1} M ; \eta, g\right)$ with the standard contact Riemannian structure. We list fundamental formulas, which are needed for the proof of our Theorem, without proofs (cf. [2], [3], [10], [28], [29]). We denote by $\nabla$ and $R$, the Levi-Civita connection and the Riemannian curvature tensor associated with $g$, respectively.

$$
\begin{aligned}
\nabla_{X^{T}} Y^{T} & =-G(Y, u) X^{T}, \\
\nabla_{X^{T}} Y^{H} & =1 / 2(K(u, X) Y)^{H}, \\
\nabla_{X^{H}} Y^{T} & =\left(\nabla_{X} Y\right)^{T}+1 / 2(K(u, Y) X)^{H}, \\
\nabla_{X^{H}} Y^{H} & =\left(\nabla_{X} Y\right)^{H}-1 / 2(K(X, Y) u)^{T},
\end{aligned}
$$

and

$$
\begin{aligned}
R\left(X^{T}, Y^{H}\right) Z^{H}= & -1 / 2\{K(Y, Z)(X-G(X, u) u)\}^{T} \\
& +1 / 4\{R(Y, K(u, X) Z) u\}^{T}-1 / 2\left\{\left(D_{Y} K\right)(u, X) Z\right\}^{H}, \\
R\left(X^{H}, Y^{H}\right) Z^{H}= & (K(X, Y) Z)^{H}+1 / 2\{K(u, K(X, Y) u) Z\}^{H} \\
& -1 / 4\{K(u, K(Y, Z) u) X-K(u, K(Y, Z) u) Y\}^{H} \\
& +1 / 2\left\{\left(D_{Z} K\right)(X, Y) u\right\}^{T}
\end{aligned}
$$

for all vector fields $X, Y$ and $Z$ on $M$, where we denote by $D$ and $K$, the Levi-Civita connection and the Riemannian curvature tensor associated with $G$, respectively. From (4.1) and (4.2), we have

$$
\nabla_{X^{T}} \xi=-2 \phi X^{T}-\left(K_{u} X\right)^{H}, \nabla_{X^{H}} \xi=-\left(K_{u} X\right)^{H},
$$

where $K_{u}=K(\cdot, u) u$ is the Jacobi operator associated with the unit vector $u$. From (2.4) and (4.4), it follows that

$$
\begin{aligned}
& h X^{T}=X^{T}-\left(K_{u} X\right)^{T} \\
& h X^{H}=-X^{H}+1 / 2 G(X, u) \xi+\left(K_{u} X\right)^{H} .
\end{aligned}
$$

Using the formula (4.3), we get

$$
\begin{aligned}
& R_{\xi} X^{T}=\left(K_{u}^{2} X\right)^{T}+2\left(K_{u}^{\prime} X\right)^{H} \\
& R_{\xi} X^{H}=4\left(K_{u} X\right)^{H}-3\left(K_{u}^{2} X\right)^{H}+2\left(K_{u}^{\prime} X\right)^{T},
\end{aligned}
$$

where $K^{\prime}=\left(D_{u} K\right)(\cdot, u) u$ and $K^{2}=K(K(\cdot, u) u, u) u$. By using (2.7), (4.1) and (4.3) we obtain

$$
\begin{aligned}
& \left(\nabla_{\xi} h\right) X^{T}=-2\left(K_{u} X\right)^{H}+2\left(K_{u}^{2} X\right)^{H}-2\left(K_{u}^{\prime} X\right)^{T}, \\
& \left(\nabla_{\xi} h\right) X^{H}=-2\left(K_{u} X\right)^{T}+2\left(K_{u}^{2} X\right)^{T}+2\left(K_{u}^{\prime} X\right)^{H} .
\end{aligned}
$$

The above formulae (4.4)-(4.7) are also found in [10]. Finally, from (4.2) and (4.6) we compute

$$
\begin{aligned}
& R_{\xi}^{\prime} X^{T}=4\left(K_{u}^{\prime} K_{u} X+K_{u} K_{u}^{\prime} X\right)^{T}+4\left(K_{u}^{\prime \prime} X+K_{u}^{2} X-K_{u}^{3} X\right)^{H} \\
& R_{\xi}^{\prime} X^{H}=8\left(K_{u}^{\prime} X-K_{u}^{\prime} K_{u} X-K_{u} K_{u}^{\prime} X\right)^{H}+4\left(K_{u}^{\prime \prime} X+K_{u}^{2} X-K_{u}^{3} X\right)^{T}
\end{aligned}
$$


Now, we prove Theorem B. Suppose that $T_{1} M=\left(T_{1} M ; \eta, g\right)$ with the standard contact Riemannian structure satisfies the condition (C) for any $\hat{\nabla}$-geodesic $\gamma$. Since the geodesic flow vector field $\xi$ also determine $\hat{\nabla}$-geodesic flow, we consider the condition

$$
\left(\hat{\nabla}_{\xi} R\right)(\cdot, \xi) \xi=0
$$

Then from (2.10) and (4.9), we see that (4.9) is equivalent to the condition

$$
R_{\xi}^{\prime}=R_{\xi} \phi-\phi R_{\xi}
$$

From (4.10), together with the (4.1), (4.6) and (4.8), we have

$$
\begin{gathered}
\left(K_{u}^{\prime} K_{u} X+K_{u} K_{u}^{\prime} X\right)^{T}+\left(K_{u}^{\prime} X\right)^{T}=0, \\
\left(K_{u}^{3} X-K_{u} X-K_{u}^{\prime \prime} X\right)^{T}=0,
\end{gathered}
$$

and

$$
\begin{gathered}
2\left(K_{u}^{\prime} K_{u} X+K_{u} K_{u}^{\prime} X\right)^{H}-\left(K_{u}^{\prime} X\right)^{H}=0, \\
\left(K_{u}^{3} X-K_{u} X-K_{u}^{\prime \prime} X\right)^{H}=0 .
\end{gathered}
$$

On the other hand, differentiating (2.6) covariantly with respect to $\xi$, then taking account of (4.10) we have

$$
\left(\nabla_{\xi} h\right) h+h\left(\nabla_{\xi} h\right)=0
$$

From (4.5), (4.7) and (4.13), we obtain

$$
\begin{gathered}
\left(K_{u}^{\prime} K_{u} X+K_{u} K_{u}^{\prime} X\right)^{T}-2\left(K_{u}^{\prime} X\right)^{T}=0 \\
\text { and }\left(K_{u}^{\prime} K_{u} X+K_{u} K_{u}^{\prime} X\right)^{H}-2\left(K_{u}^{\prime} X\right)^{H}=0,
\end{gathered}
$$

hence it follows that

$$
K_{u}^{\prime} K_{u} X+K_{u} K_{u}^{\prime} X-2 K_{u}^{\prime} X=0 .
$$

Thus, from (4.11) and (4.12), together with (4.14) we have

$$
K_{u}^{\prime} X=0
$$

and

$$
K_{u}^{3} X-K_{u} X=0
$$

for all vector field $X$ on $M$. Here, we note that $M$ satisfies the condition (4.15) if and only if $M$ is locally symmetric (see [15], [31]). Further, from (4.16) we see that the eigenvalues of $K_{u}$ are constant and $-1,0$ or 1 , that is, $M$ is a globally Osserman space (i.e., the eigenvalues of $K_{u}$ depend neither on the point $p$ nor on the choice of $u$ ). But, we know that a locally symmetric globally Osserman space is locally flat or locally isometric to rank one symmetric space (cf. [14]). Thus, we see that the base manifold $M$ is a space of constant curvature $c=1,0$ or -1 . As we have mentioned before, the unit tangent sphere bundle is a $(k, \mu)$-space if and only if the base manifold is of constant curvature $c$ with $k=c(2-c)$ and $\mu=-2 c$. Therefore, together with Theorem A, we have proved Theorem B. 
5. Three dimensional contact Riemannian manifolds. In this section we prove Theorem $\mathrm{C}$ and Theorem D. It was proved in [27] that a 3-dimensional contact Riemannian manifold always satisfies the condition $Q=0$, i.e.

$$
\left(\nabla_{X} \phi\right) Y=g(X+h X, Y) \xi-\eta(Y)(X+h X)
$$

From (2.8) and (5.1) we have

$$
R(X, Y) \xi=\eta(Y)(X+h X)-\eta(X)(Y+h Y)+\phi\left(\left(\nabla_{Y} h\right) X-\left(\nabla_{X} h\right) Y\right)
$$

for all vector fields $X$ and $Y$. We have already noted that a K-contact manifold is characterized by the condition $h=0$ and it is easily seen and well-known that a 3dimensional K-contact manifold is Sasakian. Hence, we have

Lemma 5.1. A 3-dimensional contact Riemannian manifold is Sasakian if and only if $h=0$.

Let $\left(M^{3} ; \eta, g\right)$ be a 3-dimensional contact Riemannian manifold satisfying the condition (C) for any $\hat{\nabla}$-geodesic $\gamma$. It is well-known that the curvature tensor $R$ of a 3-dimensional Riemannian manifold is expressed by

$$
\begin{aligned}
R(Y, X) Z= & \rho(X, Z) Y-\rho(Y, Z) X+g(X, Z) S Y-g(Y, Z) S X \\
& -\frac{1}{2} \tau\{g(X, Z) Y-g(Y, Z) X\}
\end{aligned}
$$

for all vector fields $X, Y, Z$, where $\rho(Y, X)=g(S Y, X)$ and $\tau$ is the scalar curvature of the manifold. From (5.3) and the assumption we have

$$
\begin{aligned}
0= & \left(\hat{\nabla}_{x} R\right)(y, x) x \\
= & \left(\hat{\nabla}_{x} \rho\right)(x, x) y-\left(\hat{\nabla}_{x} \rho\right)(y, x) x+g(x, x)\left(\hat{\nabla}_{x} S\right) y-g(y, x)\left(\hat{\nabla}_{x} S\right) x \\
& -\frac{1}{2}(x \tau)\{g(x, x) y-g(y, x) x\},
\end{aligned}
$$

for any $x, y \in T_{p} M$ and any $p \in M$. For any unit $v$ orthogonal to $\xi$, let $\{v, \phi v, \xi\}$ be an adapted orthonormal basis of $T_{p} M(p \in M)$. Then from (4.4) we get $g\left(\left(\hat{\nabla}_{x} R\right)(v, x) x, v\right)=0, g\left(\left(\hat{\nabla}_{x} R\right)(\phi v, x) x, \phi v\right)=0$ and $g\left(\left(\hat{\nabla}_{x} R\right)(\xi, x) x, \xi\right)=0$, and summing up these three equalities, we have

$$
\left(\hat{\nabla}_{x} \rho\right)(x, x)=0
$$

Also, from (5.4) we get $\left(\hat{\nabla}_{v} R\right)(\phi v, v) v=0,\left(\hat{\nabla}_{v} R\right)(\xi, v) v=0$ and thus we have

$$
\left(\hat{\nabla}_{v} \rho\right)(\phi v, \phi v)=\left(\hat{\nabla}_{v} \rho\right)(\xi, \xi)
$$

From (2.12), we have

$$
A(x, y)=\eta(x) \phi y+\eta(y)(\phi x+\phi h x)-g(\phi x+\phi h x, y) \xi
$$

for $x, y \in T_{p} M$ and $p \in M$. From (2.11) and (5.7) we have the formulas (5.8) and (5.9) 
which are equivalent to (5.5) and (5.6), respectively:

$$
\begin{gathered}
\left(\nabla_{x} \rho\right)(x, x)=4 \eta(x) \rho(\phi x, x)+2\{\eta(x) \rho(\phi h x, x)-\eta(S x) g(\phi h x, x)\}, \\
\left(\nabla_{v} \rho\right)(\xi, \xi)-\left(\nabla_{v} \rho\right)(\phi v, \phi v)=2\{(2+g(h v, v)) \rho(\xi, \phi v)+\rho(\phi h v, \xi)\},
\end{gathered}
$$

for any unit $x \in T_{p} M$ and unit vector $v$ orthogonal to $\xi$.

Let $W$ be the subset of $M$ on which the number of distinct eigenvalues of $h$ is constant. Then $W$ is an open and dense subset of $M$. We fix any point $q$ in $W$. Then, from (2.3), there exists a $C^{\infty}$ function $\lambda$ and a local orthonormal frame field $\left\{e_{1}, e_{2}=\phi e_{1}, e_{3}=\xi\right\}$ on a neighborhood $N(q)(\subset W)$ containing $q$ such that $h e_{1}=\lambda e_{1}$, $h e_{2}=-\lambda e_{2}, h \xi=0$. We denote $\Gamma_{i j k}=g\left(\nabla_{e_{i}} e_{j}, e_{k}\right), \rho_{i j}=\rho\left(e_{i}, e_{j}\right), \nabla_{i} \rho_{j k}=\left(\nabla_{e_{i}} \rho\right)\left(e_{j}, e_{k}\right)$ and $\nabla_{h} R_{i j k l}=g\left(\left(\nabla_{h} R\right)\left(e_{i}, e_{j}\right) e_{k}, e_{l}\right)$ for $h, i, j, k, l=1,2,3$. Then we get at once

$$
\Gamma_{i j j}=0 \text {. }
$$

Further from (2.4) we get

$$
\Gamma_{132}=-\Gamma_{123}=-(1+\lambda), \quad \Gamma_{231}=-\Gamma_{213}=1-\lambda
$$

and

$$
\Gamma_{113}=\Gamma_{223}=\Gamma_{331}=\Gamma_{332}=0 .
$$

Also, from (2.7) and taking account of (2.5) and (5.2), we have

$$
\xi \lambda=\rho_{12}
$$

and

$$
4 \lambda \Gamma_{312}=\rho_{22}-\rho_{11}
$$

Moreover, from (5.8) we get

$$
\nabla_{1} \rho_{11}=0, \quad \nabla_{2} \rho_{22}=0
$$

and

$$
\nabla_{3} \rho_{33}=0
$$

Differentiating (2.5) covariantly in the direction $\xi$ and taking account of (5.15) we obtain that $\xi \lambda=0$. Thus, from (5.12) we have

$$
\rho_{12}=0 .
$$

If we substitute $x=\frac{1}{\sqrt{2}}\left(e_{1}+e_{2}\right)$ and $x=\frac{1}{\sqrt{2}}\left(e_{1}-e_{2}\right)$, respectively in (5.8) and take account of (5.14), we have

$$
2 \nabla_{1} \rho_{12}+2 \nabla_{2} \rho_{12}+\nabla_{1} \rho_{22}+\nabla_{2} \rho_{11}=-4 \lambda\left(\rho_{31}+\rho_{32}\right)
$$

and

$$
-2 \nabla_{1} \rho_{12}+2 \nabla_{2} \rho_{12}+\nabla_{1} \rho_{22}-\nabla_{2} \rho_{11}=4 \lambda\left(\rho_{31}-\rho_{32}\right)
$$


By summing these two equalities, we have

$$
\nabla_{1} \rho_{22}+2 \nabla_{2} \rho_{12}=-4 \lambda \rho_{23}
$$

and subtracting from the preceding one, we have

$$
\nabla_{2} \rho_{11}+2 \nabla_{1} \rho_{12}=-4 \lambda \rho_{13} .
$$

If we substitute $x=\frac{1}{\sqrt{2}}\left(e_{1}+e_{3}\right)$ and $x=\frac{1}{\sqrt{2}}\left(e_{1}-e_{3}\right)$, respectively in (5.8) and take account of (5.15) and (5.16), we have

$$
2 \nabla_{1} \rho_{13}+2 \nabla_{3} \rho_{31}+\nabla_{1} \rho_{33}+\nabla_{3} \rho_{11}=2(\lambda+2) \rho_{23}
$$

and

$$
-2 \nabla_{1} \rho_{13}+2 \nabla_{3} \rho_{31}+\nabla_{1} \rho_{33}-\nabla_{3} \rho_{11}=2(\lambda+2) \rho_{23} .
$$

Summing these two equalities we have

$$
\nabla_{1} \rho_{33}+2 \nabla_{3} \rho_{13}=2(\lambda+2) \rho_{23} .
$$

A similar calculation for $x=\frac{1}{\sqrt{2}}\left(e_{2}+e_{3}\right)$ and $x=\frac{1}{\sqrt{2}}\left(e_{2}-e_{3}\right)$ gives

$$
\nabla_{2} \rho_{33}+2 \nabla_{3} \rho_{23}=2(\lambda-2) \rho_{13} .
$$

On the one hand, by applying the second Bianchi identity in (5.3), then taking account of (5.14) we have

$$
\begin{aligned}
& 2 \nabla_{2} \rho_{12}+2 \nabla_{3} \rho_{13}-\nabla_{1} \rho_{22}-\nabla_{1} \rho_{33}=0 . \\
& 2 \nabla_{1} \rho_{21}+2 \nabla_{3} \rho_{23}-\nabla_{2} \rho_{11}-\nabla_{2} \rho_{33}=0 .
\end{aligned}
$$

From (5.17), (5.19) and (5.21) (resp. (5.18), (5.21) and (5.24)), we have (5.23) (resp. (5.24)):

$$
\begin{aligned}
& \nabla_{1} \rho_{22}+\nabla_{1} \rho_{33}=-(\lambda-2) \rho_{23}, \\
& \nabla_{2} \rho_{11}+\nabla_{2} \rho_{33}=-(\lambda+2) \rho_{13} .
\end{aligned}
$$

On the other hand, from (5.9) we have

$$
\nabla_{1} \rho_{33}-\nabla_{1} \rho_{22}=4(\lambda+1) \rho_{23}
$$

and

$$
\nabla_{2} \rho_{33}-\nabla_{2} \rho_{11}=4(\lambda-1) \rho_{13}
$$

Thus, from (5.23)-(5.26) we have

$$
\nabla_{1} \rho_{33}=\frac{3}{2}(\lambda+2) \rho_{23}, \quad \nabla_{2} \rho_{33}=\frac{3}{2}(\lambda-2) \rho_{13}
$$

and

$$
\nabla_{1} \rho_{22}=-\frac{1}{2}(5 \lambda+2) \rho_{23}, \quad \nabla_{2} \rho_{11}=-\frac{1}{2}(5 \lambda-2) \rho_{13}
$$


Also, from (5.17), (5.18) and (5.28), we have

$$
\nabla_{1} \rho_{12}=-\frac{1}{4}(3 \lambda+2) \rho_{13} \quad \text { and } \quad \nabla_{2} \rho_{21}=-\frac{1}{4}(3 \lambda-2) \rho_{23} .
$$

Differentiating (2.5) covariantly in the directions $e_{1}$ and $e_{2}$ and taking account of (5.10), (5.11) and (5.27), we have

$$
(\lambda-2) \rho_{23}=8 \lambda\left(e_{1} \lambda\right)
$$

and

$$
(\lambda+2) \rho_{13}=8 \lambda\left(e_{2} \lambda\right)
$$

respectively.

If we also differentiate (5.16) covariantly in the direction $\xi$, then we have

$$
\nabla_{3} \rho_{12}=\Gamma_{312}\left(\rho_{11}-\rho_{22}\right) \text {. }
$$

Substituting $x=\xi$ in (5.4), we get $\hat{\nabla}_{3} \rho_{12}=0$, and from (5.7) we get $\hat{\nabla}_{3} \rho_{12}=\nabla_{3} \rho_{12}+$ $\rho_{11}-\rho_{22}$. Thus, we obtain

$$
\nabla_{3} \rho_{12}=\rho_{22}-\rho_{11}
$$

We prove

\section{LEMMA 5.2. $\lambda$ is locally constant.}

Proof. We set $N(q)=N^{0}(q) \cup N^{1}(q)$, where $N^{0}=\left\{p \in N(q) \mid \rho_{11}(p) \neq \rho_{22}(p)\right\}$ and $N^{1}=\left\{p \in N(q) \mid \rho_{11}(p)=\rho_{22}(p)\right\}$. We divide our arguments into three cases: (i) $N=N^{0}$, (ii) $N=N^{1}$ or (iii) $N^{0}$ and $N^{1}$ are both non-empty. (i) $N=N^{0}$. Then $\rho_{11} \neq \rho_{22}$ on $N$, and from (5.33) we get $\Gamma_{312}=-1$ on $N$. Thus (5.13) becomes $4 \lambda=\rho_{11}-\rho_{22}$ on $N$. Differentiating this covariantly in the directions $e_{1}$ and $e_{2}$ and taking account of (5.10) and (5.11), we have $\nabla_{1} \rho_{22}=-2(\lambda+1) \rho_{23}-4\left(e_{1} \lambda\right)$ and $\nabla_{2} \rho_{11}=-2(\lambda-1) \rho_{13}+$ $4\left(e_{2} \lambda\right)$. Thus taking account of (5.28), we have

$$
\begin{aligned}
& (\lambda-2) \rho_{23}=8\left(e_{1} \lambda\right), \\
& -(\lambda+2) \rho_{13}=8\left(e_{2} \lambda\right)
\end{aligned}
$$

on $N$. So, from (5.30), (5.31) and (5.34) we have

$$
\begin{gathered}
(\lambda-1)\left(e_{1} \lambda\right)=0, \\
(\lambda+1)\left(e_{2} \lambda\right)=0 .
\end{gathered}
$$

From (5.35) we see that $\lambda$ is locally constant on $N$. We consider the case (ii) $N=$ $N^{1}$. Then $\rho_{11}=\rho_{22}$ on $N$. Differentiating $\rho_{12}=0$ covariantly in the direction $e_{1}$ and $e_{2}$, then from the assumption, (5.10) and (5.11), we have $\nabla_{1} \rho_{12}=-(1+\lambda) \rho_{13}$ and $\nabla_{2} \rho_{21}=(1-\lambda) \rho_{23}$, respectively. Thus from (5.29) taking account of (5.30) and (5.31), we have

$$
\begin{aligned}
& (\lambda+2) \rho_{13}=0, \\
& (\lambda-2) \rho_{23}=0 .
\end{aligned}
$$


So, from (5.30), (5.31) and (5.36) we have

$$
\begin{aligned}
& \lambda\left(e_{1} \lambda\right)=0, \\
& \lambda\left(e_{2} \lambda\right)=0 .
\end{aligned}
$$

From (5.37) we see that $\lambda$ is locally constant on $N$. In case (iii), that is, $N^{0}$ and $N^{1}$ are non-empty, then in view of the above cases (i) and (ii) and by using a continuity argument, we see that $\lambda$ is constant.

Thus, from (5.30) and (5.31) we have

$$
(\lambda-2) \rho_{23}=0 \text { and }(\lambda+2) \rho_{13}=0 .
$$

If $\lambda=0$, then by Lemma $4.1 M$ is a Sasakian manifold, and further by Theorem 2.3 we see that $M$ is locally $\phi$-symmetric. From now, we suppose that $\lambda \neq 0$ and we argue by three cases: (I) $\lambda \neq \pm 2$, (II) $\lambda=2$ and (III) $\lambda=-2$.

(I) $\lambda \neq \pm 2$. Then from (5.38) we get

$$
\rho_{13}=\rho_{23}=0
$$

which yield $R\left(e_{1}, e_{2}\right) \xi=0$. Hence by using (5.2), we have

$$
\lambda\left(\Gamma_{212} e_{1}-\Gamma_{121} e_{2}\right)=0,
$$

which gives

$$
\Gamma_{212}=\Gamma_{221}=\Gamma_{121}=\Gamma_{112}=0 .
$$

Since $\lambda$ is constant, we see that $\rho_{11}=\rho_{22}$ at each point on $N$ or $\rho_{11}-\rho_{22}=4 \lambda$ at each point on $N$. We first suppose that $\rho_{11}=\rho_{22}$ on $N$. Then from (5.13) we get $\Gamma_{312}=0$. Thus, together with (5.10), (5.11) and (5.39), we have

$$
\left[e_{1}, e_{2}\right]=2 e_{3}, \quad\left[e_{2}, e_{3}\right]=(1-\lambda) e_{1}, \quad\left[e_{3}, e_{1}\right]=(1+\lambda) e_{2} .
$$

By virtue of a well-known result of the theory of Lie groups (see [30, Proposition 1.9]) and with the help of J. Milnor's classification for 3-dimensional unimodular Lie groups ([18]), we see that $M$ is locally isometric to one of the following spaces:

(i) $S U(2)$ (or $S O(3)$ ) with a left-invariant metric when $0<\lambda<1$;

(ii) $S L(2, \mathbb{R})($ or $O(1,2)$ ) with a left-invariant metric when $\lambda>1$;

(iii) flat when $\lambda=1$. In fact, if $\lambda=1$, then from (5.10), (5.11), (5.39) and (5.40) we see that $R=0$.

Next, we suppose that $\rho_{11}-\rho_{22}=4 \lambda$ on $N$. Then from (5.13) we get $\Gamma_{312}=-1$. Thus, together with (5.10), (5.11) and (5.39), we have

$$
\left[e_{1}, e_{2}\right]=2 e_{3}, \quad\left[e_{2}, e_{3}\right]=-\lambda e_{1}, \quad\left[e_{3}, e_{1}\right]=\lambda e_{2} .
$$

By similar arguments as in the former case, we see that $M$ is locally isometric to $S L(2, \mathbb{R})$ (or $O(1,2))$ with a special left-invariant metric.

If we differentiate (5.16) covariantly in the directions $e_{1}$ and $e_{2}$, then we have

$$
\begin{aligned}
& \nabla_{1} \rho_{12}=\Gamma_{121}\left(\rho_{22}-\rho_{11}\right)-(1+\lambda) \rho_{13}, \\
& \nabla_{2} \rho_{12}=\Gamma_{212}\left(\rho_{11}-\rho_{22}\right)+(1-\lambda) \rho_{23} .
\end{aligned}
$$


Together with (5.29), we have

$$
4 \Gamma_{112}\left(\rho_{11}-\rho_{22}\right)=(\lambda+2) \rho_{13} \text { and } 4 \Gamma_{221}\left(\rho_{22}-\rho_{11}\right)=(\lambda-2) \rho_{23} .
$$

We now consider the case (II) $\lambda=2$. Then from (5.38) we get $\rho_{13}=0$. Differentiating this covariantly in the directions $e_{2}$ and $e_{3}$, we obtain

$$
\nabla_{2} \rho_{13}=(1-\lambda)\left(\rho_{11}-\rho_{33}\right) \text { and } \nabla_{3} \rho_{13}=-\Gamma_{312} \rho_{23} \text {. }
$$

From (5.19), (5.27) and (5.43) we have

$$
\left(\Gamma_{312}+1\right) \rho_{23}=0 .
$$

If there are interior points where $\rho_{23}=0$, then in the same way as the case (I) we see that $M$ is locally isometric to $S L(2, \mathbb{R})($ or $O(1,2)$ ) with a special left-invariant metric. So, we restrict ourselves to the place $\Gamma_{312}=-1$. Then from (5.13) we get $\rho_{11}-\rho_{22}=8$. Hence from (5.42) we have $\Gamma_{212}=\Gamma_{221}=\Gamma_{121}=\Gamma_{112}=0$, and thus we have the same conclusion.

Finally, we consider the case (III) $\lambda=-2$. From (5.28) we get $\rho_{23}=0$. Differentiating this covariantly along $e_{1}$ and $e_{3}$, we obtain

$$
\nabla_{1} \rho_{23}=(1+\lambda)\left(\rho_{22}-\rho_{33}\right) \text { and } \nabla_{3} \rho_{23}=\Gamma_{312} \rho_{13} .
$$

From (5.20), (5.27) and (5.44) we have

$$
\left(\Gamma_{312}+1\right) \rho_{13}=0 .
$$

In a similar way as in $\lambda=2$ we see that $M$ is locally isometric to $\operatorname{SL}(2, \mathbb{R})($ or $O(1,2))$ with a special left-invariant metric. We note that $\rho_{11} \neq \rho_{22}$ in the case $\lambda=-2$.

Conversely, by Theorem 2.3 we see that a Sasakian locally $\phi$-symmetric space satisfies the condition (C) for any $\hat{\nabla}$-geodesic $\gamma$. Also, we easily see that a locally flat manifold always satisfies the condition (C) for any $\hat{\nabla}$-geodesic $\gamma$. Now, we consider a 3-dimensional Lie group with the Lie algebra structure

$$
\left[e_{1}, e_{2}\right]=c_{1} e_{3},\left[e_{2}, e_{3}\right]=c_{2} e_{1},\left[e_{3}, e_{1}\right]=c_{3} e_{2},
$$

for some constants $c_{1}(\neq 0), c_{2}, c_{3}$. Let $\left\{\omega_{i}\right\}$ be the dual 1-forms of the vector fields $\left\{e_{i}\right\}$. By using (5.45) we get $d \omega_{3}\left(e_{1}, e_{2}\right)=-d \omega_{3}\left(e_{2}, e_{1}\right)=-\frac{c_{1}}{2}$ and $d \omega_{3}\left(e_{i}, e_{j}\right)=0$ for $(i, j) \neq(1,2),(2,1)$. Further we easily check that $\omega_{3} \wedge d \omega_{3}\left(e_{1}, e_{2}, e_{3}\right)=-\frac{c_{1}}{6}(\neq 0)$, and hence $\omega_{3}$ is a contact form and $e_{3}$ is the characteristic vector field. Define a Riemannian metric $g$ and a $(1,1)$-tensor field $\phi$ by

$$
g\left(e_{i}, e_{j}\right)=\delta_{i j}, \quad d \omega_{3}\left(e_{i}, e_{j}\right)=g\left(e_{i}, \phi e_{j}\right)
$$

for $i, j=1,2,3$. Then, since $\left(\phi, \omega_{3}, g\right)$ has to be a contact Riemannian structure, we must have $g\left(\phi e_{i}, \phi e_{j}\right)=g\left(e_{i}, e_{j}\right)-\omega\left(e_{i}\right) \omega\left(e_{j}\right)$ for $i, j=1,2,3$, and hence we have $c_{1}=2$.

We recall the Koszul formula

$$
\begin{aligned}
2 g\left(\nabla_{X} Y, Z\right)= & X g(Y, Z)+Y g(Z, X)-Z g(X, Y) \\
& +g(Y,[Z, X])+g(Z,[X, Y])-g(X,[Y, Z])
\end{aligned}
$$


where $X, Y, Z$ are smooth vector fields on the manifold. Then we have

$$
\begin{gathered}
\nabla_{e_{1}} e_{1}=0, \quad \nabla_{e_{2}} e_{2}=0, \quad \nabla_{e_{3}} e_{3}=0, \quad \nabla_{e_{3}} e_{1}=\frac{1}{2}\left(c_{2}+c_{3}-2\right) e_{2}, \\
\nabla_{e_{1}} e_{3}=\frac{1}{2}\left(c_{2}-c_{3}-2\right) e_{2}, \quad \nabla_{e_{2}} e_{3}=\frac{1}{2}\left(2+c_{2}-c_{3}\right) e_{1} .
\end{gathered}
$$

Further, from (5.46) and by the definition of the curvature tensor $R$, we can compute $R\left(e_{i}, e_{j}\right) e_{k}(i, j, k=1,2,3)$. In particular, we get

$$
\begin{aligned}
R\left(e_{1}, e_{2}\right) e_{1} & =-\frac{1}{4}\left\{c_{3}^{2}+c_{2}^{2}-2 c_{2} c_{3}+4\left(c_{2}+c_{3}\right)-12\right\} e_{2}, \\
R\left(e_{1}, e_{3}\right) e_{1} & =\frac{1}{4}\left\{3 c_{3}^{2}-c_{2}^{2}-2 c_{2} c_{3}+4\left(c_{2}-c_{3}\right)-4\right\} e_{3}, \\
R\left(e_{2}, e_{3}\right) e_{2} & =\frac{1}{4}\left\{3 c_{2}^{2}-c_{3}^{2}-2 c_{2} c_{3}+4\left(c_{3}-c_{2}\right)-4\right\} e_{3}, \\
R\left(e_{1}, e_{3}\right) e_{3} & =-\frac{1}{4}\left\{3 c_{3}^{2}-c_{2}^{2}-2 c_{2} c_{3}+4\left(c_{2}-c_{3}\right)-4\right\} e_{1}, \\
R\left(e_{2}, e_{3}\right) e_{3} & =-\frac{1}{4}\left\{3 c_{2}^{2}-c_{3}^{2}-2 c_{2} c_{3}+4\left(c_{3}-c_{2}\right)-4\right\} e_{2}, \\
R\left(e_{i}, e_{j}\right) e_{k} & =0 \text { for } i \neq j \neq k \neq i,
\end{aligned}
$$

etc.

From (2.4) and (5.46) we obtain

$$
h e_{1}=\frac{c_{3}-c_{2}}{2} e_{1}, \quad h e_{2}=-\frac{c_{3}-c_{2}}{2} e_{2} .
$$

Also, from (2.11) and (5.48) we have

$$
\hat{\nabla}_{e_{3}} e_{1}=\frac{1}{2}\left(c_{2}+c_{3}\right) e_{2}, \quad \hat{\nabla}_{e_{3}} e_{2}=-\frac{1}{2}\left(c_{2}+c_{3}\right) e_{1}, \quad \text { all other } \hat{\nabla}_{e_{i}} e_{j}=0
$$

In view of (5.40) and (5.41), we consider the two possible cases: (i) $c_{2}+c_{3}=0$, (ii) $c_{2}+c_{3}=2$.

Case (i). From (5.49) it follows that $\hat{\nabla}_{e_{i}} e_{j}=0$ for $i, j=1,2,3$. Thus we see that $\hat{\nabla} R=0$.

Case (ii). Then from (5.47) we get

$$
\begin{aligned}
& R\left(e_{1}, e_{2}\right) e_{1}=\left(1-\left(c_{2}-1\right)^{2}\right) e_{2}, \\
& R\left(e_{1}, e_{3}\right) e_{1}=R\left(e_{2}, e_{3}\right) e_{2}=c_{2}\left(c_{2}-2\right) e_{3}, \\
& R\left(e_{1}, e_{3}\right) e_{3}=-c_{2}\left(c_{2}-2\right) e_{1}, R\left(e_{2}, e_{3}\right) e_{3}=-c_{2}\left(c_{2}-2\right) e_{2}, \\
& R\left(e_{i}, e_{j}\right) e_{k}=0 \text { for } i \neq j \neq k \neq i, \\
& \text { etc. }
\end{aligned}
$$

After some long but straightforward computations, we can check that the manifold satisfies $\left(\hat{\nabla}_{x} R\right)(y, x) x=0$ for all tangent vector $x$ and $y$. 
Acknowledgements. The authors wish to express their gratitude to Prof. L. Vanhecke for helpful comments and remarks in preparing this article. Also, they thank the referee for useful advice and suggestions of some references in the final version.

\section{REFERENCES}

1. E. Barletta and S. Dragomir, Differential equations on contact Riemannian manifolds, Ann. Scuola Norm. Pisa, Cl. Sci. (4) 30 (2001), 63-95.

2. D. E. Blair, Riemannian geometry of contact and symplectic manifolds, Progress in Mathematics 203 (Birkhäuser, 2003).

3. D. E. Blair, When is the tangent sphere bundle locally symmetric?, in Geometry and topology (ed. G. Stratopoulos and G. M. Rassias), (World Scientific, Singapore, 1989), 15-30.

4. D. E. Blair, Critical associated metrics on contact manifolds III, J. Austral. Math. Soc. Ser. A 50 (1991), 189-196.

5. D. E. Blair, T. Koufogiorgos and R. Sharma, A classification of 3-dimensional contact metric manifolds with $Q \phi=\phi Q$, Kodai Math. J. 13 (1990), 391-401.

6. D. E. Blair, T. Koufogiorgos and B. J. Papantoniou, Contact metric manifolds satisfying a nullity condition, Israel J. Math. 91 (1995), 189-214.

7. D. E. Blair and L. Vanhecke, Symmetries and $\phi$-symmetric spaces, Tôhoku Math. J. 39 (1987), 373-383.

8. E. Boeckx, A class of locally $\phi$-symmetric contact metric spaces, Arch. Math. (Basel) 72 (1999), 466-472.

9. E. Boeckx, A full classification of contact metric $(k, \mu)$-spaces, Illinois J. Math. 44 (2000), 212-219.

10. E. Boeckx and L. Vanhecke, Characteristic reflections on unit tangent sphere bundles, Houston J. Math. 23 (1997), 427-448.

11. E. Boeckx, D. Perrone and L. Vanhecke, Unit tangent sphere bundles and two-point homogeneous spaces, Period. Math. Hungar. 36 (1998), 79-95.

12. J. T. Cho, A new class of contact Riemannian manifolds, Israel J. Math 109 (1999), 299-318.

13. P. Dombroski, On the geometry of tangent bundle, J. Reine Angew. Math. 210 (1962), 73-88.

14. P. Gilkey, A. Swann and L. Vanhecke, Isoparametric geodesic spheres and a conjecture of Osserman concerning the Jacobi operator, Quart. J. Math. Oxford Ser. (2) 46 (1995), 299-320.

15. A. Gray, Classification des variétés approximativement kählériennes de courbure sectionelle holomorphe constante, J. Reine Angew. Math. 279 (1974), 797-800.

16. S. Ianuş, Sulle varietà di Cauchy-Riemann, Rend. Accad. Sci. Fis. Mat. Napoli (4) 39 (1972), 191-195.

17. O. Kowalski, Curvature of the induced Riemannian metric of tangent bundle of a Riemannian manifold, J. Reine Angew. Math. 250 (1971), 124-129.

18. J. Milnor, Curvature of left invariant metrics on Lie groups, Adv. in Math. 21 (1976).

19. M. Okumura, Some remarks on spaces with a certain contact structure, Tôhoku Math. J. 14 (1962), 135-145.

20. D. Perrone, Torsion tensor and critical metrics on contact $(2 \mathrm{n}+1)$-manifolds, Monatsh. Math. 114 (1992), 245-259.

21. D. Perrone, Tangent sphere bundles satisfying $\nabla_{\xi} \tau=0$, J. Geom. 49 (1994), 178-188.

22. S. Sasaki and Y. Hatakeyama, On differentiable manifolds with certain structures which are close related to almost contact structure II, Tôhoku Math. J. 13 (1961), 281-294.

23. S. Sasaki and Y. Hatakeyama, On differentiable manifolds with contact metric structure, J. Math. Soc. Japan 14 (1962), 249-271.

24. T. Takahashi, Sasakian $\phi$-symmetric spaces, Tôhoku Math. J. 29 (1977), 91-113.

25. N. Tanaka, On non-degenerate real hypersurfaces, graded Lie algebras and Cartan connections, Japan J. Math. 2 (1976), 131-190.

26. S. Tanno, The topology of contact Riemannian manifolds, Illinois J. Math. 12 (1968), $700-717$. 
27. S. Tanno, Variational problems on contact Riemannian manifolds, Trans. Amer. Math. Soc. 314 (1989), 349-379.

28. S. Tanno, The standard CR structure on the unit tangent sphere bundle, Tôhoku Math. J. 44 (1992), 349-379.

29. Y. Tashiro, On contact structures of unit tangent sphere bundles, Tôhoku Math. J. 21 (1969), 117-143.

30. F. Tricerri and L.Vanhecke, Homogeneous structures on Riemannian manifolds, London Math. Soc. Lecture Notes No 83 (Cambridge University Press, 1983).

31. L. Vanhecke and T. J. Willmore, Interactions of tubes and spheres, Math. Anal. 263 (1983), 31-42.

32. K. Yano and S. Ishihara, Tangent and cotangent bundles (M. Dekker Inc., 1973). 